\title{
La destruction et l'origine : Ernst Jünger et Walter Benjamin
}

\section{Bernd Stiegler}

\section{Abstract}

Destruction and Origin: Ernst Jünger and Walter Benjamin.

Destruction in Jünger and Benjamin is not only the mark of an anti-bourgeois stance, but a figure of integration and constructionboth a technique and a goal, the locus of transformation through a search for the origin.

\section{Citer ce document / Cite this document :}

Stiegler Bernd. La destruction et l'origine : Ernst Jünger et Walter Benjamin. In: Littérature, n¹12, 1998. La littérature et la danse. pp. 112-126;

doi : https://doi.org/10.3406/litt.1998.1606

https://www.persee.fr/doc/litt_0047-4800_1998_num_112_4_1606

Fichier pdf généré le 02/05/2018

\section{BY: $\$$ creative}




\section{La destruction et l'origine : Ernst Jünger et Walter Benjamin}

Paris, 27 mai 1944

Alertes, passages d'avions. Du toit du Raphaël j'ai vu par deux fois, en direction de Saint-Germain, s'élever d'énormes nuages, à la suite d'explosions, tandis que les flottes aériennes s'éloignaient à grande hauteur. Le but du bombardement était le pont de la Seine. La nature et la succession des opérations dirigées contre les lignes de ravitaillement dénotent un esprit subtil. La seconde fois, tandis que le soleil se couchait, je tenais à la main un verre de bourgogne où flottaient des fraises. La ville, avec ses toits et ses coupoles rougis par le couchant, s'étendait dans sa beauté puissante, tel un calice de fleur, survolé en vue d'une fécondation mortelle. Tout était spectacle, pur déploiement de pouvoir, confirmé et sublimé par la souffrance (1).

Quelques années avant cette observation de Jünger dans son Journal, Walter Benjamin note dans le cahier N des « Passages parisiens » : « La construction présuppose la destruction » (2) et reprend ainsi une réflexion assez énigmatique de son essai sur Karl Kraus écrit en 1930. Dans le texte consacré à Kraus, la destruction est à la fois un travail du texte et de son analyse.

Seul le désespéré trouve la force dans la citation : non pas la force de conserver, mais de purifier, d'arracher du contexte, de détruire; elle est la seule force recelant encore l'espoir que quelque chose de cette époque survivra - justement parce qu'on le lui a extirpé (3).

Benjamin et Jünger découvrent la force féconde de la destruction et trouvent en elle la durée et la survie. La destruction apparaît comme spectacle et travail textuel, comme procédé et fait historique, comme guerre, disparition et source messianique. La destruction est une figure, un mouvement de la réflexion et elle occupe une position centrale dans les théories philosophiques et littéraires des années 20 et 30 (4). Ce n'est pas seulement dans le combat contre la bour-

I E. Jünger, Strablungen II, München, 1965, p. $264 f$ (p. $293 \mathrm{f}$ de la traduction française).

2 W. Benjamin, Das Passagenwerk, Frankfurt/Main, 1982, p. 587. 
geoisie que se rejoignent les prises de position théoriques de Benjamin et de Jünger. La pensée de la destruction qui leur est commune se révélera comme une figure de l'intégration. La destruction est à la fois une opération et un objet qui permet la mise en relation et l'échange des éléments hétérogènes. La destruction est un espace neutre de métamorphose et de médiation. Elle apparaît toujours à l'horizon d'une recherche de l'origine.

À la recherche de l'origine perdue, la destruction est accueillie chaleureusement pour défricher la tradition. Benjamin et Jünger nous proposent deux instructions théoriques sur les travaux à venir.

Chez Jünger et chez Benjamin, la destruction est immédiatement liée à une théorie du nom propre. C'est la pureté redécouverte, intacte et prometteuse du nom propre qu'on doit mettre en lumière. La destruction doit assurer le fondement des noms comme abîme. Elle est l'ouverture d'un espace originaire au milieu de la perte et de la dépropriation. Qui aurait pensé que la guerre et la destruction auraient trouvé leur « obscur objet du désir » dans les noms propres?

Dans les théories à analyser, la destruction, l'origine et les noms propres sont intensément liés. L'espace ouvert par la destruction permet non seulement l'échange des éléments hétérogènes, mais il met en rapport des positions opposées. C'est par une affinité élective entre adversaires que Benjamin et Jünger s'approchent. Il nous faudra analyser leur rapprochement et leur séparation.

\section{ERNST JÜNGER : L'INDESTRUCTIBLE AU-DELÀ DE LA DESTRUCTION}

Pendant la guerre, Ernst Jünger se promène. Le 19 juillet 1940, après avoir rêvé de Carl Schmitt pendant la nuit, il observe les destructions dans le parc du château de Montmirail et y fait une découverte d'une signification immense :

J'étais descendu dans le parc [de Montmirail] et voulant emporter un souvenir de ce séjour, j'explorai les cratères blancs sur la pelouse, à la recherche d'éclats de bombes. Dans le premier entonnoir, j'eus la surprise d'une trouvaille beaucoup plus rare: l'admirable pétrification d'un coquillage que l'explosion avait arraché aux profondeurs de son lit de craie. (...) Moi que la destruction avait accompagné tous ces jours-ci comme une ombre, je compris aussitôt la leçon de cette découverte : le Montmirail a été jadis un récif dans la mer de craie et en elle repose, indestructible, l'élément merveilleux dont ce château lui-même, avec ses jardins, n'est qu'une émanation, une parabole éphémère, à l'instar de ce coquillage (5). 
Le souvenir de ces journées fait place à la découverte de l'indestructible de tous les jours; des éclats de bombes céderont aux traces d'une mer originaire que Jünger a déjà mise au jour et traversée pendant la Première Guerre mondiale. En 1917, il écrit à son frère Georg Friedrich :

Le service dans les tranchées est rendu plus agréable par le fait qu'on trouve dans la pierre calcaire de nombreux oursins, coquillages et ammonites. Ainsi, je me promène sur le fond des mers disparues et souvent je m'arrête devant un épaulement comme si c'était un rébus (6).

Maintenant, grâce à la réapparition de ce regard et des formes immuables de la nature, il est capable de déchiffrer le rébus: ce qui se montre par la destruction et à travers elle, c'est l'indestructible (7). Mais ce qui peut être mis au jour par les signes de la destruction n'est pas seulement le passé, mais aussi l'origine et l'avenir. Le fond de la mer originaire desséchée, c'est l'indestructible, la forme sensible de la post-histoire comme histoire originaire. L'indestructible est déchiffrable à travers les formes des animaux disparus qui, dans les coquillages, produisent leur survie de leur vivant même. On sait découvrir l'indestructible dans les formes naturelles. L'indestructible est la force formatrice de la forme donnée. La forme de l'avenir et du passé, c'est-à-dire la forme absolue, se dessine à l'horizon de la destruction (8). Dans la description du limaçon, Jünger transgresse la perception sensible de la chose pour atteindre quelque chose de suprasensible qui est en même temps représenté comme le fondement (Grund), ou plutôt l'abîme (Abgrund), à la fois de la chose et de celui qui la regarde. Le texte «L'indestructible», paru en 1930 dans la revue Das Reich ( Le royaume »), qui n'a pas été repris dans l'édition des œuvres dites complètes, est d'une clarté extraordinaire sur ce point :

La connaissance la plus précieuse qu'on tire de l'école de la guerre, est celle-ci : dans sun cueur le plus intime, la vie est totalement indestructible. Cette connaissance est donnée à tous les peuples fondamentalement guerriers, et la destruction violente précisément de la forme visible de la vie, du corps dans la bataille était considérée comme l'accès le plus certain à un pays impérissable ( $(\boldsymbol{)}$ ).

La guerre révèle la vie juste et authentique ; la guerre est elle-même la vie comme force élémentaire. Elle découvre l'autre côté de la vie qui demeure caché dans le quotidien (10). Elle montre les formes comme des étapes vers la force formatrice de la nature et de la culture. Jünger appelle ce côté de la vie l'élémentaire et, dans la guerre, il ne voit que la naissance des forces élémentaires et destructrices. L'élémentaire, l'indestructible et la vie sont les trois composantes d'une expérience de la guerre comme machine de vie. Mais, pendant la guerre,

6 Lettre citee dapres H. Schwilk. Ernst Jünger. Leben und Werk in Texten und Bildern. Stuttgart, 1988, p. 66. Cf. E. lünger. Feuer una Blut. Hamburg. 1929 . pp. 30.96 et 100.7 Cf. I. Jünger. Serahiungen II. p. 218. 8 C. la lettre de lünger adressée a Hayn Fischer. citée dans II Schwilk. loc cit. p. 124i.

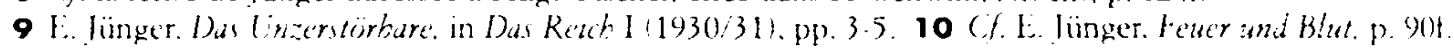


en 1940, il découvre même dans les silhouettes nocturnes de la clôture du jardin et de la haie que « les contours des formes (...) se révèlent et deviennent spirituels $\gg(11)$.

La destruction rend l'indestructible visible et imaginable; elle est l'a priori d'une pensée et d'une perception transformées (12). Elle ne change pas seulement la perception, mais aussi l'intuition intellectuelle du monde, ou bien, selon une expression de Jünger : «Chaque destruction ne fait qu'enlever l'ombre des images. (13) »Cette libération des images cachées ouvre deux perspectives: d'une part, elle produit une nouvelle perception et écriture, mais, d'autre part, elle mène à une application politique de ce qui est perçu. Cette application politique donne lieu à la théorie de la «Gestalt» (forme, figure) dans Le Travailleur. La nouvelle perception, nommée «regard stéréoscopique» dans Le Coeur aventureux (14) et «double regard» dans son essai Nationalisme et Vie moderne (15), se caractérise par une vue synesthésique des choses qui découvre en elles la surface et la profondeur en même temps. Le double regard transperce la superficie pour atteindre l'«harmonie cachée des choses 》 (16) en général dans une chose singulière. Dans un livre sur les fossiles classiques acheté sur les quais, Jünger apprend que notre limaçon a reçu son nom, Campanile giganteum, de Linné lui-même (17). Le limaçon est un signe de l'indestructible. Il témoigne, selon une note de Jünger en 1943, « d'une troisième force au-delà de la vie et de la mort » (18). Le regard stéréoscopique entre dans les choses et ouvre un abîme à la fois dans le perçu et dans le spectateur. Les coquillages ne peuvent que témoigner de l'indestructible, mais les maisons à Paris qui ressemblent à des coquillages fondent la façon de vivre de leurs habitants. La mort des habitants ayant laissé des formes vides, on peut descendre dans les maisons qui se présentent comme des formes naturelles de la culture pour y découvrir la mer originaire. Jünger dessine une paléontologie de la culture humaine en utilisant le matériau fourni par la guerre. Dans les ammonites, Jünger déchiffre des formes vides de la forme (Gestalt) de l'indestructible. Les limaçons sont des signes de la disparition anticipée de l'homme (19). Or, avant la disparition du genre humain, il faut passer par celle de l'individu dans la forme (Gestalt) du travailleur. La conception de la forme (Gestalt) met en relation les deux mouvements. L'État aussi bien que la chose naturelle sont pensés comme une forme originaire et universelle exigeant la disparition de tous ceux qui entrent en elle. La perception des formes (Gestalt) de la nature connaît une métamorphose et devient la forme (Gestalt) du travailleur comme phénomène culturel. La naissance du travailleur dans l'esprit de la guerre est en même temps la transformation de l'Histoire en histoire naturelle. Ainsi, Jünger descend dans un bunker comme si c'était un coquillage, et il y trouve «la végétation onirique intimement liée aux actes ma-

11 E. Jünger, Strablungen I, p. 127 (Jardins et Routes, p. 162).

12 Cf. E. Jünger, Der Arbeiter (= Sämtliche Werke, Stuttgart, 1962ff, vol. VIII, pp. 1-317), p. 61.

13 E. Jünger, Strahlungen I, p. 61.

14 Cf. E. Jünger, Das Abenteuerliche Herz, 2. Fassung, Frankfurt/Main, 1988, pp. 26-29.

15 Cf. E. Jünger, Nationalismus und modernes Leben, in Die Kommende 5 (1930), p. $205 f$.

16 E. Jünger, Das Abenteuerliche Herz, loc. cit, p. $29.17 \mathrm{Cf}$. E. Jünger, Strablungen II, p. 151

18 E. Jünger, Strablungen II, loc. cit., p. 30. 19 Cf. E. Jünger, Strablungen I, loc. cit., p. $145 f$.

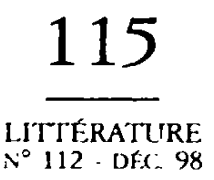


chinaux » (20). Ainsi, il tente de développer dans Le Travailleur des « concepts organiques » qui devraient déployer leur vie propre (21). La vie des concepts est due à la guerre, plus précisément à l'irruption de l'élémentaire dans le jardin de la connaissance.

On a dû plonger les racines profondément dans le sol sec pour atteindre les sources où repose l'unité magique du sang et de l'esprit, unité qui rend le mot irrésistible (22).

Pour rendre la théorie à nouveau féconde, il faut atteindre les sources de la connaissance et on les trouve dans la vie, plus précisément dans la survie, car la vie indestructible ne se montre qu'au-delà de la distinction entre la vie et la mort. Jünger voit dans la guerre

le triomphe des éléments, une irruption fulgurante de la terre elle-même, devant laquelle l'homme qui traverse les ombres en petites meutes obscures, joue un rôle minuscule et insignifiant (23).

Cette irruption de l'élémentaire dans les formes établies de l'ordre est utilisée contre la société bourgeoise. Chez Jünger, la destruction de l'ordre est basée sur un ordre de la destruction. Ce qui se dévoile dans la destruction, ce sont des formes originaires, des formes de l'origine et de l'indestructible. La destruction libère la force formatrice de la nature pour le regard, lequel répond par le langage. L'homme répond à l'activité formatrice de la vie éternelle qui crée des types (typenbildend) par les noms qui imposent des types (typensetzend). Ainsi, la dénomination répète la création dans le transitoire (24). Les noms sont des traductions de l'être impérissable qui peut être saisi dans l'effroi, dans l'anéantissement et dans la destruction où il apparaît pour un moment fugitif. Le nom et la langue ont leur origine dans la destruction. La destruction répétée rajeunit la langue et la ramène à ses sources. La figure de la destruction est une tentative de faire parler le fondement muet de toutes les apparitions, c'est-à-dire l'être. Dans ce modèle théorique, la dénomination est une réponse à une activité de la nature qui la précède. Chez Benjamin, plus précisément dans le texte de jeunesse «Sur le langage en général et sur le langage de l'homme», le nom de l'homme répond au mot divin dans les choses; chez Jünger, le nom est une réponse à l'appel de ce qui n'a pas de nom, de l'innommable. L'innommable (das Namenlose) est le fond sans fond de la culture et de la nature. Dans la guerre, la destruction de l'ennemi compte moins que celle de la société bourgeoise, de laquelle au déclin et à la destruction on peut assister. La guerre libère l'élémentaire et transforme un paysage culturel en panorama naturel. La destruction est un spectacle de la nature. 
La ville, avec ses toits et coupoles rougis par le couchant, s'étendait dans sa beauté puissante, tel un calice de fleur, survolé en vue d'une fécondation mortelle. Tout était spectacle, pur déploiement de pouvoir, confirmé et sublimé par la souffrance (25).

La beauté est la fécondation par la destruction. La destruction est la pénétration à l'intérieur de l'objet. Paris rougeoyant est prêt pour la fécondation. Les avions qui planent sur la ville comme un vol de grues (26) préparent l'entrée dans le monde caché, fermé par des verrous de feu. Leurs semences pénètrent, selon l'expression de Jünger, jusqu'au «fond originaire du sang rouge » (27) dévoilé au spectateur qui prend une jouissance à contempler la scène. La fécondation du calice par la destruction offre au voyeur non seulement l'image d'une défloration, mais aussi le spectacle d'une douleur. L'indestructible est mis au jour par la destruction et peut être nommé et ainsi possédé.

Avant la découverte du limaçon, Jünger rêvait de Carl Schmitt ; maintenant il lui adresse une lettre :

Il reste encore à Paris quelque chose d'une substance indestructible; parfois, au cours de ces semaines et de ces mois, les hommes et les choses laissent apparaittre quelque chose de presque douloureux dans une beauté significative. C'est la seule ville avec laquelle j'entretienne une liaison, comme si elle était une femme. Elle s'est comportée d'ailleurs de façon féminine en ne m'opposant aucune résistance (28).

Cette fécondation mystique à laquelle Jünger assiste avec une désinvolture presque divine ne montre point du tout l'anéantissement et la destruction conme une force opposée à l'Éros (29), mais comme Éros. C'est dans la destruction comme spectacle que son rêve d'une «grande distillation » (30) et d'une « représentation de la pierre philosophale venant des écumes basses et des levures obscures » $\left(\mathbf{3}_{1}\right)$ se réalise. La forme indestructible et la forme de l'indestructible naissent grâce à la « copulation » entre la destruction et la fécondation. Elle est la pierre philosophale, le fruit de la douleur due à la défloration de Paris pendant la guerre. Mais elle ne se révèle qu'au spectateur désintéressé qui observe la destruction comme spectacle, c'est-à-dire comme une ouvre d'art, pour la représenter ensuite dans le langage.

Après le rêve de Schmitt et la lettre qui lui est adressée, voici sa remarque féconde sur les « rapports entre la lune, les femmes et la mer » :

Les rapports étymologiques entre les coquillages et les parties génitales féminines sont très significatifs, comme par exemple, dans la parenté entre le latin conca et le danois kudefisk, où Kude a la valeur de vulve (32). 
La relation des éléments et leur fondation sans fond se montre dans la dénomination. Partant de la guerre et de la mer originaire, en passant par l'indestructible et la forme (Gestalt), la théorie de la destruction nous a menés jusqu'à Paris. C'est à Paris que la destruction rencontre la fécondation.

\section{WALTER BENJAMIN : LA DESTRUCTION DE LA DESTRUCTION OU L'ORIGINE AU-DELÀ DE LA DESTRUCTION}

La polémique signifie détruire un livre en détruisant quelques-unes de ses phrases. Moins on l'a étudié, mieux c'est. Seul celui qui peut anéantir peut critiquer. La véritable polémique s'en prend au livre aussi doucement qu'un cannibale prépare un nourisson (33).

En suivant cette affirmation de Benjamin, nous pouvons constater non seulement qu'il fut gourmand de livres, mais aussi que l'acte de lire constitue une forme du cannibalisme. Lire est une façon de déguster un livre pour le digérer très lentement. Le résultat de ce repas sera un texte qui, semble-t-il, peut également être dévoré. Alors, ce sera à moi de préparer le repas et je vous souhaite bon appétit.

Lire est le plaisir de la destruction décomposante et transformante d'un texte. Quand Benjamin, tout en rêvant d'une nouvelle barbarie, constate dans Expérience et Pauvreté que la Première Guerre mondiale avait mené à une forme radicale de la pauvreté ainsi qu'à la fin de la narration, c'est le cannibalisme de la lecture qui ouvre la voie. Le « concept nouveau et positif de la barbarie » (34) qui « commence par le commencement, commence à bâtir sur le Neuf sans regarder ni à droite ni à gauche » (35), exige un nouvel art culinaire de la préparation du livre par la lecture. On prend la Mobilisation totale de Jünger; on la met au feu sous le regard décomposant de l'analyse, puis on enlève une phrase telle que «Ceux qui sont tombés se rendaient, tandis qu'ils tombaient, d'une réalité imparfaite, de l'Allemagne de l'apparence temporelle dans l'Allemagne éternelle » (36) de sa peau textuelle pour l'examiner à la lumière. Dans la guerre, on découvrira la nature et, sur le champ de bataille, le sentiment allemand pour la nature. La contribution de Jünger à l'idylle germanique (37), c'est la transformation d'une scène de guerre en paysage naturel qui, en même temps, et en passant par la destruction, libère la vue sur le déclin de la société et l'avènement de la Gestalt (forme). Mais comment avaler ce morceau apparemment indigeste de la Mobilisation totale sans que la bouchée reste crue et coincée à mi-gorge? Comment transformer à nouveau la nature en culture transmutable?

33 W'. Benjamin, Die Technik des Kritikers in dreizehn Thesen. in Cesammelte Schriften IV (-: (iS). Frankfurt/Main, 1972ff, p. 108f, p. 108.

34 W. Benjamin, Erfabrung und Armut, GS II, pp. 213.219, p. 215 (p. 72 de la traduction française. P() E- $57 l$ : 52). 35 Ibid. $36 W^{\prime}$. Benjamin, Theorien des deutschen taschismus. (iS IJI. pp. 238-249, p. 245. 
Comme toutes les questions fondamentales de l'art culinaire, la lecture vise la différence entre le cru et le cuit, c'est-à-dire entre la nature et la culture. Les fossiles et les coquillages de Jünger sont extrêmement difficiles à préparer parce que les escargots ont déjà disparu. La lecture gourmande de Benjamin est essentiellement destructrice et poursuit la destruction du texte jüngerien. Mais, chez Benjamin, la destruction vise la construction. La lecture destructrice est l'ouverture d'un champ et d'un chantier de construction. Mais comment peut-on par la simple lecture attaquer la guerre, celle-ci étant un spectacle naturel qui libère pour le regard appropriant les forces élémentaires? Comment attaquer la guerre, comment la reprendre sans être pris par elle et sans retomber dans la soi-disant logique organique de ses concepts?

Avant tout, il faut prendre au sérieux la guerre comme une guerre de la technique. Benjamin et Jünger ont cette perspective d'analyse en commun, parce que Jünger, dans la Mobilisation totale, avait souligné le déchaînement et la surpuissance de la machine. Chez Jünger, le combat est à la fois un combat de la machine et contre la machine. Mais cette machine libère la vie, l'élémentaire et l'indestructible. C'est justement par la machine que la force de la nature renait. Chez Benjamin, la technique est considérée comme la nature des rapports sociaux qui rencontrent dans la guerre la possibilité de leur transformation essentielle. Selon Benjamin, ceci n'est possible qu'au « cours de l'exécution du truc marxiste qui seul est à la hauteur de ce sombre sortilège runique »(38). Cet aspect de la critique benjaminienne ne souligne dans la technique que sa possibilité de réconciliation de la société. Cette réception presque enthousiaste de la technique est constitutive pour pratiquement tous les textes de Benjamin dès les années 30, comme par exemple L'CEuvre d'art à l'époque de sa reproduction mécanisée ou le livre sur Baudelaire. C'est le premier côté de la reformulation d'une théorie marxiste de l'histoire. Une seconde formulation qui met l'accent sur l'origine et le nom lui correspond.

Benjamin et Jünger, le national-bolchévisme et ladite théorie marxiste de l'histoire sont d'accord sur l'évaluation des possibilités constructrices de la technique. Mais ces deux théories se séparent dans la concrétisation de cette construction.

Dans le cahier $N$ des « Passages parisiens », on trouve plusieurs réflexions qui thématisent directement le rapport entre la théorie matérialiste de l'histoire et la destruction. On peut les considérer comme des explications et des instructions concernant le «truc marxiste » pour la lecture. Critique et destruction se rencontrent si la continuité, l'homogénéité et la clôture de l'histoire éclatent.

Le moment destructeur et critique dans la conception matérialiste de l'histoire se fait valoir dans l'éclatement de cette continuité de l'histoire par laquelle l'objet historique se constitue d'abord (39). 
L'objet singulier ne peut être analysé qu'en étant enlevé de son contexte. Pour arriver à des certitudes historiques, il ne faut pas seulement abandonner la conception d'une continuité historique, c'est-à-dire qu'on doit faire le procès du procès, mais aussi modifier la perspective dans et par laquelle l'objet est visé. Dans ce contexte, Benjamin parle d'un tournant copernicien dans la conception de l'histoire qui ne comprendra plus le passé comme un ensemble monolithique, mais qui en fait l'ouverture de la conscience éveillée (40). Benjamin comprend l'étude matérialiste de l'histoire comme une décomposition des données, comme une destruction du matériau historique. L'objet de cette étude se constitue dans la destruction et apparaît en elle. Il se situe à l'intérieur de la destruction, c'est-àdire dans un horizon critique.

Il faut comprendre le moment destructeur dans la conception matérialiste de l'histoire comme une réaction à une constellation de dangers qui menace à la fois la tradition et le contenu de la tradition (41).

La destruction par la lecture est une réponse à la destruction et à la décomposition de l'objet qui est déjà à l'œuvre. La destruction opère contre la destruction et en elle. La destruction tente de détruire la destruction et de suspendre le mouvement général. Chez Benjamin, c'est un moment messianique qui remplace la paléontologie de la culture humaine chez Jünger, laquelle ne fait que mettre au jour des formes du Même, tout en associant l'origine et le telos dans une forme radicalement conservatrice. Le but d'un tel travail du texte est, selon une formule de Friedrich Engels, «de sortir du domaine de la pensée » (42). Ceci ne sera possible que si l'on cherche des ruptures inhérentes au matériau donné. La pensée occulte ces ruptures jusqu'à ce qu'apparaisse une rupture dans le mouvement de la pensée même. Seule une analyse du matériau du langage sera à même d'ouvrir cet espace inexploré. Libérer l'histoire originaire dans l'histoire, ouvrir l'origine par une lecture destructrice, faire entendre le murmure du langage pur dans le texte, voici les tâches du lecteur. Mais l'origine n'apparaît qu'en étant suspendue. D'après l'essai sur Karl Kraus, la société doit être ramenée à un état originaire qu'elle n'a jamais connu. L'origine est le moment messianique de l'histoire et de la lecture.

À la réflexion appartient le mouvement aussi bien que la suspension des pensées. C'est là où la pensée est suspendue dans une constellation saturée par des tensions que l'image dialectique apparaît. Elle est une césure dans le mouvement de la pensée. Bien sûr, sa place n'est pas une place quelconque. En un mot, elle est à chercher là où la tension entre les extrêmes dialectiques est la plus forte (43).

Benjamin nomme «image dialectique » ce qui se montre comme objet dans ce mouvement de pensée et de lecture. Si l'on applique à la théorie jüngerienne le modèle proposé pour localiser la rupture ou la césure, on peut consta-

40 W. Benjamin, Das Passagenwerk, ioc. cil., p. 490. 41 W. Benjamin, Das Passagenucrk, loc. cil., p. $594 f$. 
ter que le moment de la plus grande tension se situe entre la destruction et la fécondation, autrement dit entre Éros et Thanatos, entre la ville et l'avion, entre la civilisation et la guerre. C'est entre eux que l'indestructible, l'élémentaire et les formes originaires d'une histoire naturelle apparaissent. Le regard stéréoscopique de Jünger, capable de voir deux qualités de l'objet à la fois et de transpercer la superficie, correspond à l'image dialectique dans la conception benjaminienne. Chez Benjamin, la destruction vise la césure dans le procès intellectuel, c'est-à-dire la libération des images dialectiques qui peuvent désormais opérer comme des agents de la destruction. Dans la conception jüngerienne, la destruction enlève l'ombre des images; chez Benjamin, elle suspend la dialectique pour libérer des images. Ces images naissent de la rencontre et du choc entre le passé et le présent. Elles se présentent grâce à l'utilisation du double sens de quelques éléments du matériau donné. Les passages parisiens sont des passages dans le texte, dans la langue, des passages entre le passé et le présent, entre la réalité onirique et historique. Les passages ne sont ni des maisons ni des rues, ils ne sont ni de purs espaces intérieurs ni des espaces extérieurs. Dans l'espace historique du $\mathrm{XIX}^{\mathrm{c}}$ siècle, ils occupent une position ambivalente qui permet à la lecture de les déchiffrer comme images dialectiques. Comme dans les images stéréoscopiques de Jünger, le regard peut osciller entre l'intérieur et l'extérieur, entre le rêve et la conscience. Des images de la réalité historique se dévoilent comme de virulentes images oniriques.

Ce n'est pas que le passé jette sa lumière sur le présent ou que le présent jette sa lumière sur le passé, mais l'image est le moment où le passé se réunit comme un éclair avec le «maintenant » en une constellation. En d'autres termes : l'image est la dialectique en suspension. L'image lue, c'est-à-dire l'image dans le «maintenant » de sa perceptibilité porte en elle de façon extrême l'empreinte du moment critique et dangereux qui est à la base de toute lecture (44).

La lecture se situe dans l'horizon du danger, tout en étant elle-même un danger pour l'intégrité du texte. La lecture en danger met en danger l'ordre établi du matériau. La citation est une technique centrale de la pratique de lecture chez Benjamin. Elle est appliquée aussi bien au texte historique qu'au texte littéraire. Citer veut dire arracher un élément de son contexte, faire sauter des ensembles signifiants et utiliser stratégiquement le matériau (45). La citation est l'attaque de la lecture dans le royaume du langage.

Ce qui n'est dit que de façon allusive dans les « Passages parisiens » trouve son explication dans l'essai consacré à Karl Kraus. J'essaie de résumer en quelques traits les principaux enjeux de cette démarche, même s'il est impossible ici d'analyser ce texte très dense de façon détaillée.

Ni la pureté, ni le sacrifice n'ont asservi le démon; mais lorsque l'origine et la destruction se rencontrent, c'en est fait de sa domination. À la fois enfant et 
cannibale, son vainqueur se dresse devant lui : non pas un homme nouveau, mais un être inhumain, un ange nouveau. Peut-être de ceux qui, selon le Talmud, sont créés à chaque instant par légions innombrables pour se taire et disparaître dans le néant après avoir élevé leur voix devant Dieu. Se lamentent-ils, accusent-ils, exultent-ils ? Peu importe — l'œuvre éphémère de Kraus imite cette voix qui rapidement - s'éteint. Angelus - c'est le messager des gravures anciennes (46).

Ces lignes constituent la fin du texte, une fin qui renvoie à son début, autrement dit qui reprend le début, où le messager, tenant le journal «Fackel » (La torche) dans ses mains, est mentionné pour la première fois. Le texte entier peut être compris comme une réflexion sur le nom «Fackel» qui est à la fois nom propre et nom commun. Cette réflexion est une mise en œuvre appliquée et décisive de la destruction. La destruction s'inscrit déjà dans la dédicace hors texte, puisque Benjamin a tracé un portrait de Gustav Glück, auquel il a dédié l'essai, dans son texte «Sur le caractère destructeur » (47).

L'essai comprend trois chapitres, intitulés respectivement « L'homme universel », «Démon » et «L'inhumain ». Benjamin procède d'une façon destructrice en essayant de détruire et de purifier la démarche de Kraus en général. La fin est donc le début détruit. On trouve dans ce texte une citation de Kraus qui illustre parfaitement ce but : «Tu viens de l'origine - l'origine est la fin. (48)» Le travail critique de Benjamin vise l'origine à l'intérieur du langage - cachée et même détruite par la représentation ou l'objectivation. Kraus en tant qu'homme universel est caractérisé par son rêve d'un état à la fois paradisiaque et naturel de la société. Il parle au nom de la création pour rétablir ses lois dans la décadence et le déclin de la société. Benjamin constate chez lui une théorie réactionnaire et une pratique révolutionnaire (49). Sa démarche théorique consiste à chercher un état mythique qui devrait dominer; sa pratique, par contre, détruit l'état des faits grâce à un travail sur la citation, qui brise le contexte et la cohérence fonctionnelle de la sémantique.

Kraus, en tant que démon, apparaît dans une ambiguité fondamentale par la manière dont il essaie de critiquer, voire de détruire l'autre, sans pouvoir éviter de retomber dans le même piège. L'expression de soi et le démasquage de l'autre deviennent « auto-démasquage » (50). Kraus en tant qu'« inhumain » ne vise plus l'état présumé paradisiaque de la nature, mais cherche des voies nouvelles pour l'humanité. Cela passe par la citation qui «appelle le mot par son nom, l'arrache à son contexte en le détruisant, mais le rappelle en même temps à son origine ». Le nom est le lieu où l'origine et la destruction se rencontrent.

On touche ici au caractère éphémère de ce journal, caractère dont il a conscience dès le début. Tel est, en effet, le juste prix qu'exige sa recherche d'une actualité véritable. Car enfin, selon une légende talmudique, les anges sont bien créés - 
de nouveaux anges par légions innombrables à chaque instant - pour, après avoir chanté leur hymne devant Dieu, se taire et disparaître dans le néant. Puisse son nom signifier que ce journal acquerra une telle actualité, qui seule est vraie (51).

Ceci est la fin d'un texte programmatique de Benjamin, dans lequel il annonce la tâche, la forme et la destination de son journal - qui d'ailleurs n'a jamais paru — «Angelus Novus » (52). La réflexion sur le nom dans l'essai sur Kraus engage donc également le nom et la démarche de son propre journal. Le texte tente de repenser la fonction possible de la presse. Je résume les éléments essentiels qui débouchent aussi sur une conception du nom. La notion-clef est la citation, puisque la grande force de Kraus est d'avoir rendu le journal citable. Le journal met en rapport la sphère du public et celle du privé. Kraus, qui déteste l'opinion publique parce qu'elle appartient à la sphère privée, cherche à briser la fausse objectivité de la presse en tant qu'incarnation de l'opinion publique. La presse rend le public incapable de juger de quelque chose, parce qu'elle établit une attitude d'irresponsabilité et de non-information. Kraus, en tant que polémiste, utilise la citation pour atteindre une actualité plus fondamentale, et mène un combat titanesque contre la presse qui prépare, selon lui, la fin de l'humanité. Kraus, en cherchant l'origine naturelle, détruit l'actualité présentée par la presse et la juge à partir de la justesse/justice du langage. Il vise à suspendre l'ambiguïté mythique du droit en se rendant inhumain. La citation, comme moyen principal de la polémique, vise l'origine à l'intérieur du langage, qui ne se montre que dans le nom. Le nom est la source principale de sa lutte contre la presse. Il se soustrait dans sa valeur magique à la fausse objectivité de la presse, mais doit être mis en lumière sans pouvoir être représenté ou objectivé.

C'est dans la chambre que j'occupai en dernier lieu, avant que lui-même ne m'apparût en pleine lumière, revêtu des armes de mon ancien nom, que son image se fixa chez moi : Nouvel Ange. La Cabbale raconte qu'à chaque seconde, Dieu crée une infinité de nouveaux anges qui n'ont d'autre destin, avant de disparaître dans le néant, que de chanter sa louange un instant durant, devant son trône. Le mien avait été interrompu : ses traits n'avaient rien d'humain (53).

Ces lignes se trouvent dans un texte autobiographique de Benjamin, intitulé «Agesilaus Santander». On voit que même le nom propre de Benjamin, caché dans ce texte, est engagé - à travers une citation de la légende talmudique - dans l'essai sur Kraus. Le nom est le lieu où l'origine et la destruction s'interpénètrent. Il marque le non-lieu à l'intérieur du texte, qui n'est pas véhiculé par lui, mais qui se situe tout de mêre dans le langage et reste à découvrir par un travail critique et destructeur. Le nom appelle et rappelle l'origine, sans

51 W'. Benjamin, Ankindigung der Zeitschrift Angelus. Von us, (iS II. pp. 241 246. p. 246. 
pouvoir la nommer. Le nom en tant que «langage-du-langage » (c'est une expression de son essai sur le langage) laisse apparaitre l'essence non communicative du langage. «Citer un mot signifie l'appeler par son nom. (54)» Le travail de la citation, travail de destruction, est un appel au langage, voire au nom en tant que langue pure.

Elle n'a rien d'inepte, cette citation qui retentit, bien à sa place dans la structure d'un texte nouveau. En tant que rime, elle réunit les analogies dans son aura; en tant que nom, elle est seule et inexpressive. En elle les deux domaines - origine et destruction - se légitiment par rapport à la langue. Et inversement : ce n'est que lorsque les deux s'interpénètrent - dans la citation - que la langue s'accomplit (55).

La critique vise cette langue accomplie qui reflète la langue des anges après avoir détruit le contexte idyllique du sens. «Angelus Novus » est le nom d'un ange qui n'a rien d'humain. Son nom lie la destruction, l'origine et la fin. Tout comme la destruction critique, le mouvement de la polémique est une réappropriation de l'origine. La polémique parle au nom de la force originaire de la nature, la critique vise l'origine comme fin. Elle cherche l'origine déjà détruite pour la remettre au jour par la destruction. Dans l'essai sur Kraus et dans l'article sur Jünger, il s'agit d'une destruction au second degré, car Benjamin considère déjà la polémique chez Kraus comme une forme de destruction. Aux yeux de Benjamin, Kraus critique toujours au nom de la puissance de la nature qui entre sur la scène comme maitresse et source de la destruction. La culture est attaquée dans la perspective de la nature ; la destruction originaire de la nature est prolongée par la polémique. Chez Kraus, la polémique tente de préparer la voie pour une réintronisation imaginée de la nature. Il voit l'origine dans la nature, tandis que Benjamin tente de dépasser la distinction entre culture et nature afin d'éviter de rendre mythiques les rapports sociaux en les naturalisant.

$\mathrm{Si}$, dans la destruction de la guerre chez Jünger, Benjamin constate la métamorphose de la société en paysage naturel, cette observation vaudra également pour Kraus, parce que c'est par le regard polémique de ce dernier que la société devient nature. La fécondité de la force destructrice de la nature apparaît seulement dans la perspective de la technique introduite par Benjamin comme possibilité de réaliser la nature dans la société humaine. La création de la destruction présuppose la destruction de la création. Création et destruction s'unissent dans le langage. La citation est la technique de la destruction dans le domaine du langage.

$\mathrm{La}$ destruction vise l'origine. Le langage de l'origine est l'essence de la communication même. L'origine du langage est - selon l'essai «Sur le langage en général et le langage de l'homme » - l'immédiateté (Unmittelbarkeit) de la communicabilité du langage, ou bien, en d'autres termes, la communication 
immédiate de sa communicabilité. Dans l'essai sur Kraus, Benjamin reprend la distinction entre mot et nom de son texte dit de jeunesse. Le nom y est présenté comme l'imprégnation la plus intime entre l'appel et le dire, entre l'homme et la chose. Les noms sont le lieu dans le langage d'une relation entre le sujet et l'objet qui n'est pas encore rendue obscure par la représentation. Par les noms que l'homme donne aux choses, il répond à leur communication. Les choses appellent l'homme qui, en tant que nomothète, exprime à la fois leur essence et la sienne. Jünger comprend les noms comme un rapport entre une activité de l'homme instituant des types (typussetzend) et de la nature donnant des types (typusgebend). Dans la conception benjaminienne des noms et du langage, c'est également le rapport entre deux activités comprises comme formes de communication qui détermine leur essence. Jünger aussi bien que Benjamin tentent de libérer l'origine par la destruction. La lecture et la guerre sont les deux techniques de la destruction. Chez Benjamin, la destruction nous mène, tout en traversant la représentation, jusqu'aux noms. Chez Jünger, elle vise la forme (Gestalt) à laquelle les noms répondent. Dans les textes de Jünger on a pu suivre l'observation d'une destruction fécondante, c'est-à-dire d'une procréation; dans les textes benjaminiens, nous avons progressé d'un âge, parce que c'est dans l'enfant qu'il découvre la sensibilité pour l'origine du langage.

C'est par la rime que l'enfant reconnait qu'il a atteint la cime du langage où il perçoit le bruissement de toutes les sources dans l'origine (56).

Dans la lecture, Benjamin cherche les possibilités de faire entendre ce murmure de l'origine. La destruction des textes doit garantir la réussite de cette recherche. Elle doit transpercer et briser le langage des mots et des concepts pour atteindre les noms. La citation seule est à la hauteur de ce projet critique. Elle «appelle le mot par son nom, l'arrache de façon destructrice de son contexte et le rappelle ainsi à son origine » (57).

La destruction de la citation visc lc nom caché dans lc mot. L'cnfant ct le cannibale doivent se rencontrer afin de lier l'origine et la destruction: l'enfant qui entend le murmure du langage et le cannibale qui dévore les textes. Ils nous donnent la mesure de la destruction: «une humanité qui s'éprouve dans la destruction $\gg(\mathbf{5 8})$.

\section{LA LECTURE DEVANT LA DESTRUCTION : COM- MENT LIRE LA DESTRUCTION ?}

Si ces dispositions venaient à disparaitre comme elles sont apparues, si par quelque événement dont nous pouvons tout au plus pressentir la possibilité, mais dont nous ne connaissons pour l'instant encore ni la forme ni la promesse, (...) alors on peut bien parier que l'homme s'effacerait, comme à la limite de la mer un visage de sable (59). 
Nous avons déjà ramassé quelques coquillages sur la plage imaginée par Michel Foucault. Le mouvement de la mer désagrège le sable, formes et empreintes disparaissent. Dans l'excavation d'une bombe, Jünger découvre un coquillage fossile qui aurait pu intéresser Benjamin. Pour Jünger seule compte la forme qu'on peut retrouver dans la culture humaine. Sous le regard du théoricien de la destruction, la forme (Gestalt) du coquillage dans la nature devient rapidement la forme (Gestalt) du travailleur dans le domaine politique. Benjamin, par contre, s'intéresse plutôt au murmure originaire du langage, un murmure qui semble venir de l'intérieur du coquillage. Grâce à la lecture destructrice, il tente de faire entendre ce murmure en deçà de la signification. La forme et le langage sont des tentatives de découvrir l'origine et l'histoire originaire, la destruction est une effraction dans le jardin de la connaissance. Comme toujours, Jünger y découvre des formes éternelles, Benjamin en revanche est plutôt séduit par l'appel du langage pur des noms. Les formes permettent aussi bien que les images d'arracher des objets singuliers à leur contexte et de faire naître des constellations. Chez Benjamin et Jünger, ce ne sont pas seulement les images du rêve et de l'observation qui s'associent et se superposent, mais aussi le passé, l'avenir et le présent. Le trait destructeur de la conception matérialiste de l'histoire est structurellement apparenté au regard stéréoscopique de Jünger. L'objet de la destruction est la destruction de l'objet. La destruction vise, pour reprendre une formule de Jünger, une troisième puissance au-delà de la vie et de la mort. On peut déterminer de multiples façons ce point d'indifférence dans le mouvement général. L'origine et l'indestructible peuvent s'y nicher. Étant à la fois absent et présent, ce point d'indifférence est le centre secret des théories de Jünger et de Benjamin. La destruction déstabilise l'ordre établi, soi-disant pour le refonder de façon originaire. En ce sens, la destruction est une opération conservatrice. Jünger découvre la post-histoire comme histoire originaire, Benjamin dévoile le messianique dans le langage des noms. La destruction ouvre un espace libre. Prendre possession de cet espace signifierait retomber dans une métaphysique de l'origine.

La destruction est une technique précise permettant l'échange et le déplacement des éléments textuels. Des textes et des théories utilisent de telles zones d'échange qui ne sont pas déterminées par des lois. L'opération de la destruction ouvre des espaces d'un échange généralisé. La théorie de la destruction révèle le théorème de l'origine. Nous avons vu que des théories opposées se rencontrent dans une telle perspective. 